\title{
Protocol Development for Preventing Contrast-Induced Nephropathy
}

\author{
Rana Ibrahim*, Osama Mohamed Ibrahim \\ Department of Pharmacy Practice and Pharmacotherapeutics, College of Pharmacy \\ University of Sharjah, UAE \\ Corresponding author's email: ribrahim [AT] sharjah.ac.ae
}

\begin{abstract}
Contrast-induced nephropathy (CIN) is related to substantial economic and clinical consequences, including extended hospitalization, the necessity for dialysis, and an amplified risk of death. As radiographic diagnostic testing becomes commonplace, a significant concern among practitioners is the prevention of radiographic contrast-induced nephropathy. Radiographic contrast agents are used most often in tests such as coronary angiography, percutaneous coronary angioplasty, atherectomy, and stent placement, computer-aided tomography (CAT) scanning, and magnetic resonance imaging (MRI). 1 Administration of these contrast agents often cause acute changes in renal function ranging from an acute reduction of renal function to the transient need for hemodialysis.
\end{abstract}

Early studies assessing the renal effects of radiocontrast administration in dogs proved a reduction in renal perfusion lasting up to 20 hours after radiocontrast administration. 8 Although no randomized controlled trial (RCT) has studied the benefits of hydration alone, it appears reasonable that sufficient hydration may offset some of the presumed hemodynamic effects that could lead to contrast-induced nephropathy. Various preventative strategies have been studied with mixed results.

This paper constitutes reviewing current and old practices and developing a tentative simple protocol for prevention of Contrast induced nephropathy in nearby hospitals.

Keyworks--- Nephropathy, hydration, radiographic contrast, dialysis, renal perfusion

\section{INTRODUCTION AND BACKGROUND}

Contrast-induced nephropathy (CIN) is related to substantial economic and clinical consequences, including extended hospitalization, the necessity for dialysis, and an amplified risk of death. As radiographic diagnostic testing becomes commonplace, a significant concern among practitioners is the prevention of radiographic contrast-induced nephropathy. Radiographic contrast agents are used most often in tests such as coronary angiography, percutaneous coronary angioplasty, atherectomy, and stent placement, computer-aided tomography (CAT) scanning, and magnetic resonance imaging (MRI). ${ }^{1}$ Administration of these contrast agents often cause acute changes in renal function ranging from an acute reduction of renal function to the transient need for hemodialysis. This occurs in certain patient populations that have a preexisting renal impairment particularly when the reduction is secondary to diabetic nephropathy. Other factors in considering the moderate to high-risk patient include renal insufficiency (creatinine greater than $1.5 \mathrm{mg} / \mathrm{dl}$ ), renal surgery, proteinuria, and uncontrolled hypertension. This reduction in renal function can lead to substantial morbidity and mortality during hospitalization and has been reported to account for more than $10 \%$ of hospital-acquired renal failure. ${ }^{2,3}$

Contrast-induced nephropathy (CIN) is defined as acute renal failure occurring within 48 hours of exposure to IV contrast that is not readily attributable to other causes. The serum creatinine level begins to rise within 24 hours of administration of IV contrast. Within three to five days, this level will typically peak then return to baseline values within 10 to 14 days. Various intrinsic causes of nephrotoxicity include vasoconstriction, direct renal toxicity caused by oxygen free radicals, decreased prostaglandin and nitric oxide induced vasodilation, increased oxygen consumption, and ischemia secondary to increased interstitial pressure, increased urinary viscosity, and tubular obstruction. Extrinsic causes of nephrotoxicity can be attributed to decreased intravascular volume, diabetes, hypotension, hypertension, sepsis, additional nephrotoxic drugs, and dehydration. 
Early studies assessing the renal effects of radiocontrast administration in dogs proved a reduction in renal perfusion lasting up to 20 hours after radiocontrast administration. ${ }^{8}$ Although no randomized controlled trial (RCT) has studied the benefits of hydration alone, it appears reasonable that sufficient hydration may offset some of the presumed hemodynamic effects that could lead to contrast-induced nephropathy. Various preventative strategies have been studied with mixed results. Administration of adequate hydration appears to be the easiest method to provide a renal protective outcome. Patients at risk of developing contrast induced renal impairment have received $0.9 \%$ normal saline at 1 $\mathrm{ml} / \mathrm{kg} / \mathrm{hr}$ approximately 6-12 hours prior to the procedure with a continuous infusion for 12-24 hours after the study as compared to $0.45 \%$ sodium chloride. It was clearly found that $0.9 \%$ infusion was superior to the $0.45 \%$ normal saline group. ${ }^{4}$ The disadvantages of the volume expansion are that it is not suitable for patients with cardiac failure and is of limited use in emergency-situations where fluid administration for hours prior to the study is impractical. ${ }^{5} \mathrm{~A}$ study by Merten et al compared a one-hour pre-procedure hydration with normal saline versus sodium bicarbonate. ${ }^{3}$ Rates of contrast- induced nephropathy were lower in the sodium bicarbonate group than the patients receiving sodium chloride. However, the final results of this study are in question since the study was terminated early with the differences occurring possibly by chance. It is currently recommended that a 6-12 hour intravenous hydration regimen prior to and after radiocontrast alone or in combination with the sodium bicarbonate infusion (Sodium Bicarbonate $150 \mathrm{mEq}$ in $1000 \mathrm{ml}$ $\mathrm{D} 5 \mathrm{~W}$ at $3.5 \mathrm{ml} / \mathrm{kg} / \mathrm{hr}$ for one hour prior to contrast study followed by $1.2 \mathrm{ml} / \mathrm{kg} / \mathrm{hr}$ for six hours after contrast study completion) should be first line in preventative management of CIN.

Another method involves the use of $\mathrm{N}$-acetylcysteine for CIN prevention. $N$-acetylcysteine has been the most widely studied of all prophylaxis strategies, although the mechanism for its claimed nephroprotective action is unclear. $N$ acetylcysteine might act by scavenging oxygen free radicals ${ }^{9,10}$ or by enhancing the vasodilatory effects of nitric oxide. ${ }^{11}$ Looking to the results from 22 trials $^{12-33}$ (2918 participants) compared $N$-acetylcysteine with placebo. The results showed that relative risks for contrast-induced nephropathy ranged from 0.11 to 1.5 (median, 0.72). Eleven of 20 trials that reported contrast-induced nephropathy and 13 of 20 trials that conveyed a variation in SCr level as an outcome preferred $\mathrm{N}$-acetylcysteine prophylaxis (results from 5 studies were statistically significant).

Furthermore, the initial study on $\mathrm{N}$-acetylcysteine and radiocontrast nephropathy reported a $89 \%$ absolute risk reduction with $\mathrm{N}$-acetylcysteine. ${ }^{6}$ Many studies occurred to replicate these results with subsequent meta-analyses to follow. It was then reported that a reduction in relative risk of 0.44-0.65 occurred in favor of N-acetylcysteine with a dose of $600 \mathrm{mg}$ orally every 12 hours for 24 hours prior and post study when combined with IV hydration during a 24-hour periprocedure period together with non-ionic, low-molecular weight contrast. However, all the meta-analyses acknowledged potential flaws of study heterogeneity and publication bias. ${ }^{7}$ It is generally accepted that although $\mathrm{N}$-acetylcysteine is well tolerated and inexpensive, it is still unclear whether it significantly reduces the risk of radiocontrast-induced nephropathy. Nevertheless, the use of N-acetylcysteine is commonly employed as an option for the prevention of CIN.

Based on these current opinions, a protocol dependent on patient specific parameters is proposed for use in a hospital. (See attached Appendix I)

\section{PROPOSED PROTOCOL}

In the neuroscience Intensive Care Unit, identifying at risk patients for contrast induced nephropathy prior to CT scan or MRI is typically a hit-or-miss process. Some patients are identified while others are considered retrospectively. A process was implemented for the prescribing of agents to prevent moderate to high-risk patients from developing a contrastinduced nephropathy. The clinical NICU pharmacist will assume the responsibility of identifying, prescribing (with approval from the staff Neuro-Intensivist), and ensuring that the at-risk patient receives the CIN prophylaxis at the proper timing prior to study. During the morning rounding period, the NICU pharmacist will identify any at risk patient who will be undergoing a radiologic study with contrast dye. The pharmacist will then complete the "Contrast Nephropathy Protocol Medication Order" sheet and place it in the patient's chart. To expedite the administration of the prescribed agent, the Critical Care Pharmacy will be phoned to send the appropriate agent to the unit as soon as possible.

To assess compliance of this protocol, a list of patients who underwent a radiocontrast imaging will be obtained from the department of radiology on a daily basis. This list will be reviewed to assess whether the appropriate patients were identified, prescribed, and received prophylactic treatment prior to study initiation. This data can be further analyzed to measure compliance of this protocol.

\section{ENDPOINTS FOR PROTOCOL SUCCESS}

1) Compliance in the identification of moderate to high-risk patients for the development of contrast-induced nephropathy.

2) Compliance in the prescribing of prophylactic agents for CIN.

3) Decrease incidence of Radiocontrast nephropathy as defined as a rise of $0.5 \mathrm{mg} / \mathrm{dl}$ or $\geq 25 \%$ increase in serum creatinine over 48 hours post-procedure.

4) Appropriate timing of administration of CIN prophylactic agent (s) 
5) Decrease need for dialysis pot-procedure.

6) Decrease length of hospitalization

\section{CONCLUSION}

The discovery of novel therapies for the prevention of contrast-induced nephropathy has been hindered by an incomplete understanding of its pathophysiology. Experimental and preclinical studies must continue to be a priority for investigators operating in this field. Risk factors for contrast-induced nephropathy should be thoroughly identified and categorized into patient related and non-patient related factors, before any prophylactic treatment is initiated. Patientrelated factors include, diabetes mellitus, age, congestive heart failure, low hematocrit, hypertension, and low ejection fraction. Non-patient related factors, are related to contrast properties such as high- osmolar contrast, ionic contrast, contrast viscosity and volume. ${ }^{34,35}$

In summary, there is suggestive, but incomplete, evidence supporting the use of hydration as a prophylaxis measure for contrast-induced nephropathy. Questions persist about whether all patients benefit equally from this treatment, as well as the optimal type, route, volume, and timing of hydration administration.

\section{REFERENCES}

1. Mokhtari V, Afsharian P, et al. A Review on Various Uses of N-Acetylcysteine. Cell J. 2017;19 (1): 11-17.

2. Li Jing-Xiu, Jin En-Ze, et al. Oral N-acetylcysteine for prophylaxis of contrast-induced nephropathy in patients following coronary angioplasty: A meta-analysis. Experimental and therapeutic medicine. 2017; 14: 1568-1576.

3. Merten G, et al. Prevention of Contrast-Induced Nephropathy with Sodium Bicarbonate. A Randomized controlled Trial. JAMA. 2004; 291:2328-2334.

4. Mueller C, Buerkle G, Buettner HJ, et al. Prevention of contrast media-associated nephropathy: randomized comparison of two hydration regimens in 1620 patients undergoing coronary angioplasty. Arch Intern Med 2002; 162:329-36.

5. Morcos SK et al. Prevention of contrast media nephrotoxicity-the story so far. Clin Radiology 2004; 54:381389.

6. Tepel M van der Giet M, Schwarzfeld C, et al. Prevention of radiographic contrast-agent-induced reductions in renal fuction by acetylcysteine. N Eng J Med 2000; 343: 180-184.

7. Lin J, Bonventre JV. Prevention of radiocontrast nephropathy. Curr Opin Nephrol Hypertens 14: 105-110.

8. Goic Joana B, Koenigshof Amy M, et al. A retrospective evaluation of contrast-induced kidney injury in dogs. Journal of Veterinary Emergency and Critical Care 2016;26 (5) 713-719.

9. Meyer M, LeWinter M, Bell Stephen P, et al. N-Acetylcysteine enhanced contrast provides cardio-renal protection. JACC 2009; 2:215-221.

10. Filho Luiz D, Saad K, et al. Effect of N-acetylcysteine in hearts of rats submitted to controlled hemorrhagic shock. Braz J Cardiovasc Surg 2015;30 (2): 173-81.

11. Safirstein R, Andrade L, Vieira JM. Acetylcysteine and nephrotoxic effects of radiographic contrast agents - a new use for an old drug. N Engl J Med. 2000; 343:210-212.

12. Azmus AD, Gottschall C, Manica A, et al. Effectiveness of acetylcysteine in prevention of contrast nephropathy. J Invasive Cardiol. 2005; 17:80-84.

13. Baker CS, Wragg A, Kumar S, De Palma R, Baker LR, Knight CJ. A rapid protocol for the prevention of contrast-induced renal dysfunction: the RAPPID study. J Am Coll Cardiol. 2003; 41:2114-2118.

14. . Briguori C, Manganelli F, Scarpato P, et al. Acetylcysteine and contrast agent-associated nephrotoxicity. J Am Coll Cardiol. 2002;40:298-303.

15. Diaz-Sandoval LJ, Kosowsky BD, Losordo DW. Acetylcysteine to prevent angiography-related renal tissue injury (the APART trial). Am J Cardiol. 2002; 89: 356-358.

16. Drager LF, Andrade L, Barros de Toledo JF, Laurindo FR, Machado Cesar LA, Seguro AC. Renal effects of Nacetylcysteine in patients at risk for contrast nephropathy: decrease in oxidant stressmediated renal tubular injury. Nephrol Dial Transplant. 2004; 19:1803-1807.

17. Durham JD, Caputo C, Dokko J, et al. A randomized controlled trial of N-acetylcysteine to prevent contrast nephropathy in cardiac angiography. Kidney Int. 2002; 62:2202-2207.

18. Efrati S, Dishy V, Averbukh $\mathrm{M}$, et al. The effect of $\mathrm{N}$-acetylcysteine on renal function, nitric oxide, and oxidative stress after angiography. Kidney Int. 2003; 64:2182-2187.

19. Fung JW, Szeto CC, Chan WW, et al. Effect of N-acetylcysteine for prevention of contrast nephropathy in patients with moderate to severe renal insufficiency: a randomized trial. Am J Kidney Dis. 2004; 43: 801-808.

20. Goldenberg I, Shechter M, Matetzky S, et al.Oral acetylcysteine as an adjunct to saline hydration for the prevention of contrast-induced nephropathy following coronary angiography: a randomized controlled trial and review of the current literature. Eur Heart J. 2004; 25:212-218. 
21. Gomes VO, Poli de Figueredo CE, Caramori P, et al. N-acetylcysteine does not prevent contrast induced nephropathy after cardiac catheterisation with an ionic low osmolality contrast medium: a multicentre clinical trial. Heart. 2005; 91:774-778.

22. Gulel O, Keles T, Eraslan H, Aydogdu S, Diker E, Ulusoy V. Prophylactic acetylcysteine usage for prevention of contrast nephropathy after coronary angiography. J Cardiovasc Pharmacol. 2005; 46:464-467.

23. . Kay J, Chow WH, Chan TM, et al. Acetylcysteine for prevention of acute deterioration of renal function following elective coronary angiography and intervention: a randomized controlled trial. JAMA. 2003; 289:553558.

24. Kotlyar E, Keogh AM, Thavapalachandran S, et al. Prehydration alone is sufficient to prevent contrastinduced nephropathy after day-only angiography procedures- a randomised controlled trial. Heart Lung Circ. 2005; $14: 245-251$.

25. MacNeill BD, Harding SA, Bazari H, et al. Prophylaxis of contrast-induced nephropathy in patients undergoing coronary angiography. Catheter Cardiovasc Interv. 2003; 60:458-461.

26. Miner SE, Dzavik V, Nguyen-Ho P, et al. N-acetylcysteine reduces contrast-associated nephropathy but not clinical events during long-term follow-up. Am Heart J. 2004; 148:690-695.

27. Ochoa A, Pellizzon G, Addala S, et al. Abbreviated dosing of N-acetylcysteine prevents contrast-induced nephropathy after elective and urgent coronary angiography and intervention. J Interv Cardiol. 2004; 17:159165.

28. Oldemeyer JB, Biddle WP, Wurdeman RL, Mooss AN, Cichowski E, Hilleman DE. Acetylcysteine in the prevention of contrast-induced nephropathy after coronary angiography. Am Heart J. 2003; 146:E23.

29. Rashid ST, Salman M, Myint F, et al. Prevention of contrast-induced nephropathy in vascular patient undergoing angiography: a randomized controlled trial of intravenous N-acetylcysteine. J Vasc Surg. 2004; 40: 1136-1141.

30. Shyu KG, Cheng JJ, Kuan P. Acetylcysteine protects against acute renal damage in patients with abnormal renal function undergoing a coronary procedure. J Am Coll Cardiol. 2002; 40:1383-1388.

31. Tepel M, van der Giet M, Schwarzfeld C, Laufer U, Liermann D, Zidek W. Prevention of radiographiccontrastagent-induced reductions in renal function by acetylcysteine. $N$ Engl J Med. 2000; 343:180-184.

32. Webb JG, Pate GE, Humphries KH, et al. A randomized controlled trial of intravenous $\mathrm{N}$-acetylcysteine for the prevention of contrastinduced nephropathy after cardiac catheterization: lack of effect. Am Heart J. 2004; 148:422-429.

33. Briguori C, Colombo A, Violante A, et al. Standard vs double dose of N-acetylcysteine to prevent contrast agent associated nephrotoxicity. Eur Heart J. 2004; 25:206-211.

34. Aspelin P, Aubry P, Fransson SG, Strasser R,Willenbrock R, Berg KJ. Nephrotoxic effects in high-risk patients undergoing angiography.NEngl JMed. 2003; 348:491-499.

35. Andreucci M, Faga T, Serra R, et al. Update on the renal toxicity of iodinated contrast drugs used in clinical medicine. Drug Healthc Patient Saf. 2017; 9:25-37. 


\section{Appendix I: CONTRAST NEPHROPATHY PROTCOL MEDICATION ORDER}

DATE:

PATIENT NAME:

\section{ASSESS PATIENT FOR CONTRAST NEPHROPATHY RISK FACTORS}

Serum creatinine $\geq 1.5 \mathrm{mg} / \mathrm{dl}$, diabetes, volume depletion, concurrent use of the following medications: NSAIDS, ACE Inhibitors, Angiotensin Receptor Blocker, Diuretics

\section{HYDRATION CONTRAINDICATED:}

\section{(Volume overload or severe heart failure/LV dysfunction)}

- $\quad \mathrm{N}$-acetylcysteine $600 \mathrm{mg}$ orally every 12 hours x 4 doses

$>$ Initiate 24 hours prior to study or as soon as possible

\section{HYDRATION INDICATED:}

(Radiologic procedure will be performed within 6 hours)

- N-acetylcysteine $600 \mathrm{mg}$ orally every 12 hours x 4 doses

$>$ Initiate 24 hours prior to study or as soon as possible

\section{CHOOSE HYDRATION REGIMEN:}

- Sodium Chloride $0.9 \% 500 \mathrm{ml}$ infusion over -hours (choose 1-6 hours) prior to contrast study followed by Sodium Chloride $0.9 \% 500 \mathrm{ml}$ infusion over 2 hours after contrast study completion

\section{OR}

- Sodium Bicarbonate $150 \mathrm{mEq}$ in $1000 \mathrm{ml} \mathrm{D} 5 \mathrm{~W}$ at $3.5 \mathrm{ml} / \mathrm{kg} / \mathrm{hr}$ for one hour prior to contrast study followed by $1.2 \mathrm{ml} / \mathrm{kg} / \mathrm{hr}$ for six hours after contrast study completion

Prescribing Physician Signature: 